\title{
NATIVE VASCULAR FLORA OF BEHALI RESERVE FOREST (ASSAM, INDIA) WITH GLOBAL IUCN RED LIST ASSESSMENT OF TWO ENDEMIC SPECIES
}

\author{
Dipankar BORAH ${ }^{1,2,3}$, Sumpam TANGJANG ${ }^{2}$ Abhaya P. DAS $S^{2}$ \\ Parixit KAFLEY $Y^{3}$, Anatoliy A. KHAPUGIN ${ }^{4,5}$ \\ ${ }^{1}$ Department of Botany, Goalpara College, Goalpara-783101, Assam, India \\ ${ }^{2}$ Department of Botany, Rajiv Gandhi University, Rono Hills, Doimukh-791109, Arunachal Pradesh, India \\ ${ }^{3}$ Nature's Bonyopran, Behali, Biswanath-784176, Assam, India \\ ${ }^{4}$ Joint Directorate of the Mordovia State Nature Reserve and National Park "Smolny", Krasnaya Street, 30 \\ Saransk-430005, Republic of Mordovia, Russia \\ ${ }^{5}$ Institute of Environmental and Agricultural Biology (X-BIO), Tyumen State University, Lenina Street, 25, \\ 625003-Tyumen, Russia \\ e-mail: sumpam@gmail.com
}

\begin{abstract}
The investigations of flora and fauna in Protected Areas provide important contributions to the knowledge on the status of unmanaged natural ecosystems. In 2017-2019, we studied the vascular plant flora of Behali Reserve Forest (Assam, India). The reserve forest covers $140.16 \mathrm{~km}^{2}$ area of semi-evergreen forest. We investigated the taxonomic composition of the flora and diversity of growth forms of species and habitats. In addition, we conducted global IUCN Red List assessment of two endemic species, Aristolochia assamica and Chlorophytum assamicum. We found that the vascular plant flora of Behali Reserve Forest consists of 281 taxa, including 272 species, one subspecies and eight varieties. They belong to 206 genera and 79 families. Among them, Pandanus unguifer was recorded for the first time for the flora of Assam. In growth form spectrum of vascular plants, trees, vines and shrubs predominated, followed by epiphytes, forbs or herbs, geophytes, annuals and parasites. The largest number of vascular plants was found in dense forests, while open habitats were characterized by the smallest species richness. Chlorophytum assamicum is assessed as CR B1ab(iii)+2ab(iii); D, and Aristolochia assamica as EN B2ab(iii). At the same time, there is a threat of reduction or disappearance of their populations. This is especially crucial for $C$. assamicum, represented by a single world population. Undoubtedly, further investigations of flora could result in an increase in number of the vascular plant flora of Behali Reserve Forest, as well as providing essential insights into population trends of several other species as per IUCN Red List categories and criteria.
\end{abstract}

Keywords: angiosperms, checklist, foothills, Himalayas, Protected Area, threat status.

\section{Introduction}

About $15 \%$ of tropical forests play the primary role of biodiversity protection [40] and cover $40 \%$ of the world's wooded areas. Similarly, these tropical forests harbour a considerable proportion of global biodiversity [17, 47, 14, 30]. They occupy about $48,295 \mathrm{~km}^{2}(7.72 \%)$ of the forests of India [42]. The state of Assam comprises diverse vegetation types, hosting a rich biodiversity. Apart from the tropical semi-evergreen forests, several other forest types such as deciduous forests, evergreen forests and swamp forests are prevalent in the region. The Flora of Assam [29] still acts as a comprehensive account for the vascular plants of the entire Northeastern India. However, later it was supplemented by several sporadic publications [16, 37, 3, 2], but there is still a lack of accurate data on plant diversity from numerous sub-regions of Assam, including the Protected Areas. 
At present, Protected Areas are considered to be the main tool for in situ conservation and restoration $[21,28]$ of biodiversity around the world. In India, the Protected Area network covers $5.02 \%$ of the whole geographical area of country, including 104 national parks, 551 wildlife sanctuaries, 88 conservation reserves and 127 community reserves [35]. Although there are several publications on the biodiversity of Protected Areas in Assam, the understanding of its status and diversity still remains incomplete [36]. Among the Protected Areas network, Reserve Forests are the least protected and are generally under very high anthropogenic pressure. So far, little is known about the biological diversity of these Reserve Forests in India, including in the state of Assam. To date, the biodiversity of the several Protected Areas of India is underestimated and such is the case of Behali Reserve Forest (BRF). Recently, three new taxa, Chlorophytum assamicum D.Borah \& A.P.Das, Aristolochia assamica D. Borah \& T.V. Do, and Peliosanthes macrophylla var. assamensis N.Tanaka \& D.Borah, were described from BRF and Tupistra stoliczkana Kurz was re-discovered after more than a century $[8,9,10,11]$. In addition, the existence of a wild Citrus indica Yu.Tanaka population has been reported in this Protected Area, although it was previously reported to be extinct in the state of Assam [7]. These reports underpin the need for study of these reserve forests and for analysis of their role in protection of several important and endangered biodiversity elements.

The present study was aimed to study the vascular flora of BRF with special focus on accessing global IUCN Red List status for two endemic plant species.

\section{Material and Methods}

\section{Study area}

BRF is located in the outermost foothills of the Eastern Himalaya (Fig. 1). It is a part of the Kameng-Sonitpur Elephant Reserve that also includes other Protected Areas of Assam (Balipara Reserve Forest, Charduar Reserve Forest, Nowduar Reserve Forests, Nameri Tiger Reserve) and Arunachal Pradesh (Eagle Nest Wildlife Sanctuary, Pakke Tiger Reserve, Sessa Orchid Sanctuary and Reserve Forests of Khellong Forest Division, Sonai-Rupai Wildlife Sanctuary) [31]. Together with the Kaziranga National Park on its south, BRF acts as a corridor for different animals to the highlands during floods. So far very few floristic studies have been conducted in the Biswanath district $[15,4,45]$ and a general account on the local biodiversity of BRF have been published [44] with no mention of its unique flora.

The area of BRF covers $140.16 \mathrm{~km}^{2}$ of semi-evergreen forest [46], that falls under the Biswanath district of Assam state, India sharing its boundaries with Buroi River in the East, Borgang river and Singlijan Reserve Forest in the West, Papum Reserve forest in the North, and tea plantations and human habitations in the South [44]. It comprises mostly of plains between $26.866667^{\circ}-26.950000^{\circ} \mathrm{N}$ and $93.250000^{\circ}-93.416667^{\circ} \mathrm{E}$ and a series of hills on its extreme north. The mean annual temperature is $24.8^{\circ} \mathrm{C}$ and mean annual precipitation is $1800 \mathrm{~mm}$. The altitude ranges between $90 \mathrm{~m}$ a.s.1. to $110 \mathrm{~m}$ a.s.1. [44]. 


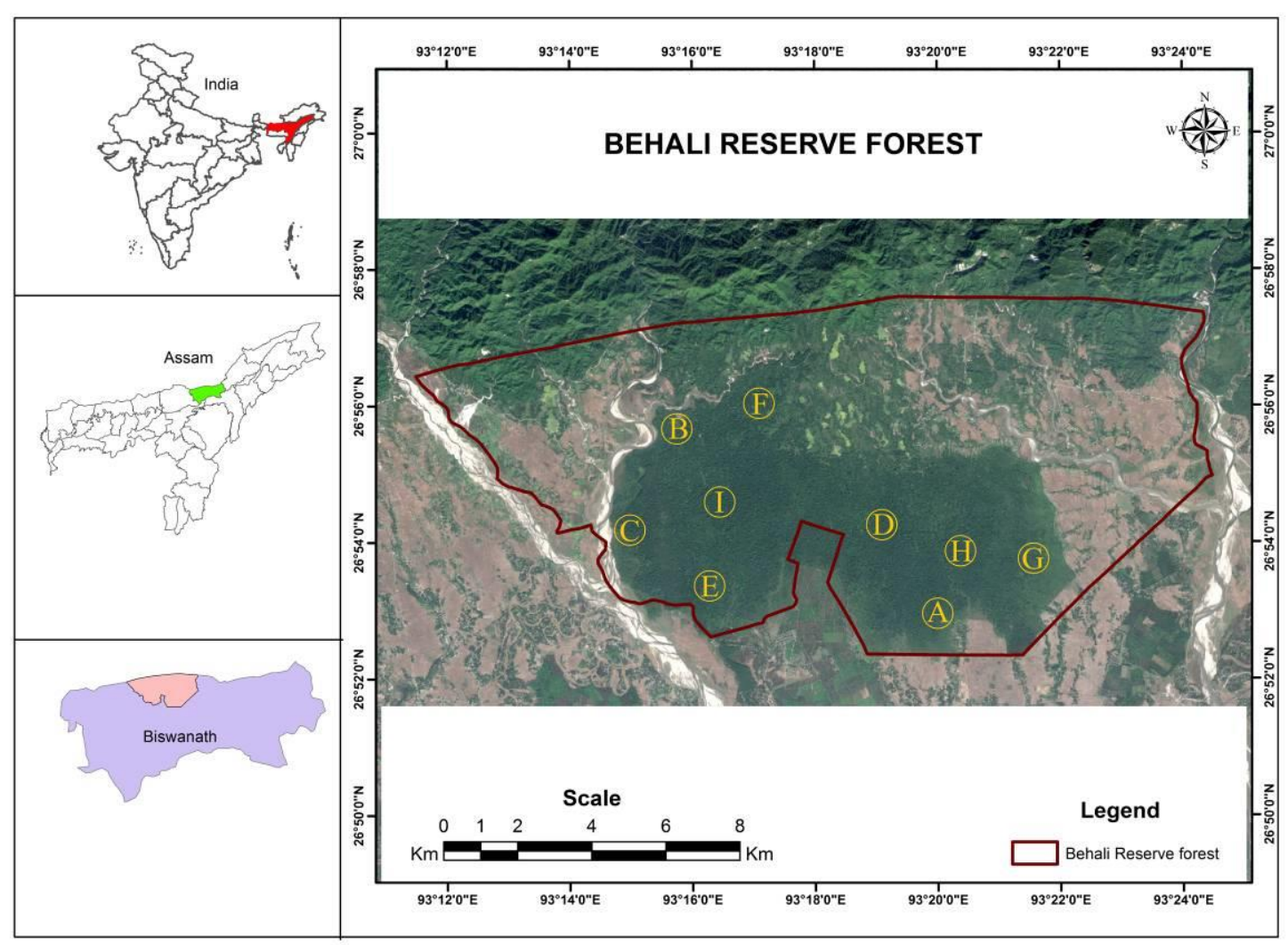

Fig. 1: Map of Behali Reserve Forest and main study sites (defined by letters).

\section{Floristic data sampling and analysis}

Extensive floristic surveys were conducted from April 2017 to August 2019. In total, nine study sites were sampled during the field surveys (Fig. 1, Table 1). The Latin names of plants are based on the database POWO [41]. Collected herbarium specimens were identified using available determinant keys $[22,29]$. The confusing taxa were identified with the help of taxonomic group specialists. The voucher specimens collected were later processed and mounted into herbarium sheets following the methods of Jain \& Rao [27], and then deposited in HAU (Herbarium of Rajiv Gandhi University, India).

Table 1: Description of the study sites in the Behali Reserve Forest.

\begin{tabular}{c|l|l|l}
\hline Site & \multicolumn{1}{|c|}{ Dates of the surveys } & \multicolumn{1}{|c}{ Protected Area; study site location } & \multicolumn{1}{c}{ Co-ordinates; altitude } \\
\hline A & July 2017; April 2019 & Behali Reserve Forest; Bongaon & $26.882361^{\circ} \mathrm{N}, 93.333528^{\circ} \mathrm{E} ; 103 \mathrm{~m}$ a.s.l. \\
\hline B & June 2018; April 2019 & Behali Reserve Forest; Dikal & $26.926944^{\circ} \mathrm{N}, 93.267917^{\circ} \mathrm{E} ; 98 \mathrm{~m}$ a.s.l. \\
\hline C & April 2017; August 2018 & Behali Reserve Forest; Hatidipu & $26.900306^{\circ} \mathrm{N}, 93.253389^{\circ} \mathrm{E} ; 101 \mathrm{~m}$ a.s.l. \\
\hline D & March 2018; August 2019 & Behali Reserve Forest; Hatimara & $26.902472^{\circ} \mathrm{N}, 93.322944^{\circ} \mathrm{E} ; 97 \mathrm{~m} \mathrm{a.s.1.}$ \\
\hline E & April 2018; June 2019 & $\begin{array}{l}\text { Behali Reserve Forest; Rangagorha } \\
\text { Beat }\end{array}$ & $26.886722^{\circ} \mathrm{N}, 93.267111^{\circ} \mathrm{E} ; 92 \mathrm{~m}$ a.s.l. \\
\hline F & June 2017; July 2019 & Behali Reserve Forest; Radhasu & $26.929583^{\circ} \mathrm{N}, 93.281417^{\circ} \mathrm{E} ; 95 \mathrm{~m} \mathrm{a.s.1.}$ \\
\hline G & July 2018; April 2019 & Behali Reserve Forest; Sialmari & $26.895389^{\circ} \mathrm{N}, 93.362028^{\circ} \mathrm{E} ; 104 \mathrm{~m}$ a.s.l. \\
\hline H & April 2017; April 2019 & Behali Reserve Forest; Serelia & $26.896972^{\circ} \mathrm{N}, 93.342167^{\circ} \mathrm{E} ; 101 \mathrm{~m}$ a.s.l. \\
\hline I & March 2018; August 2019 & $\begin{array}{l}\text { Behali Reserve Forest; Siklibandha } \\
\text { Tiniali }\end{array}$ & $26.906639^{\circ} \mathrm{N}, 93.272417^{\circ} \mathrm{N} ; 99 \mathrm{~m}$ a.s.l. \\
\hline
\end{tabular}

To characterize the studied flora, we estimated growth forms of all species and the habitat 
types where plants were found. Growth forms were characterised according to IUCN Plant Growth Forms Classification Scheme [24]. To describe habitat diversity, we used the following definitions. 1) Pristine forest (PF): unmanaged forest patches with both well-developed canopy and three-tier vegetation. 2) Secondary forests (SF): forest patches affected by anthropogenic deforestation, that was resulted in both forest stand and canopy reduction making the illumination intensity as relatively high. 3) Rehabilitated forests (RF) are a result of restoration of a degraded forest to its original state by planting native trees. 4) Shrublands (SH): shrubby plant community characterized by vegetation dominated by shrubs with participation of grasses, herbs, and geophytes. 5) Riparian habitats (RH) are considered as areas along the banks of a permanent or ephemeral water body (river, stream, spring or waterfall). 6) Grasslands (GR) are open habitats dominated mainly by grasses, sedges, rushes, being dry or flooded seasonally. The global IUCN Red List categories of each species were indicated following IUCN [26].

\section{IUCN Red List assessment}

Assessments of the conservation status of two endemic species, Chlorophytum assamicum and Aristolochia assamica, for the IUCN Red List followed the recently revised evaluation criteria adopted by IUCN [23, 25], if any one of the IUCN Red List criteria is met. As we have no detailed and reliable population-level data for these plant species, species conservation assessments have been based on IUCN criteria B [13]: an evaluation of range size measured as either extent of occurrence (EOO, criterion B1) or area of occupancy (AOO, criterion B2) followed by an assessment of population fragmentation. In addition, we used criterion $\mathrm{D}$, as an indicator of a very small or restricted population. The extent of occurrence (EOO) and area of occupancy (AOO) were calculated using GeoCAT tool [1]. To make our assessment in line of high-quality global studies, we used recent publications devoted to global and sub-global IUCN assessments of vascular plants $[38,20,32]$.

\section{Results}

\section{Flora of vascular plants in the Behali Reserve Forest}

The flora of Assam state consists of a total 2823 vascular plants which belong to 309 genera, and 107 families [29]. However, in a relatively small area of BRF, 281 vascular plant species are reported, which is equal to about $10 \%$ of the erstwhile Assam flora.

During field expeditions, about 347 herbarium specimens were collected in the different vegetation types and communities. They represent 281 taxa (to simplify, hereafter - «species»), including 272 species, one subspecies and eight varieties of vascular plants, which belong to 206 genera and 79 families (Table 2). Of these taxa, Pandanus unguifer Hook. f. (Fig. 2) is recorded here as a first report for the flora of Assam.

To date, P. unguifer was known from South Sikkim district of Central Himalaya and Darjeeling district of upper West Bengal [49]. This species is closely related to the recently described P. martinianus Nadaf \& Zanan. It can be distinguished by its globular syncarp with 90110 randomly distributed drupes (vs. ellipsoid syncarp with 140-160 drupes arranged in rows in P. martinianus) [48]. Specimen examined: India, Assam, Behali Reserve Forest, Siklibandha Tiniali 2654'23.9" N, 9316'20.7" E, 99 m a.s.1., 12.03.2018, D. Borah (BRF785) (HAU). 
Table 2: Native vascular flora of Behali Reserve Forest (Assam, India).

$$
\text { Family }
$$

Taxon

Fabaceae

Orchidaceae

Orchidaceae

Lamiaceae

Lauraceae

Orchidaceae

Gesneriaceae

Meliaceae

Sapindaceae

Zingiberaceae

Zingiberaceae

Lauraceae

Lauraceae

Primulaceae

Araceae

Araceae

Phyllanthaceae

Phyllanthaceae

Phyllanthaceae

\section{Meliaceae}

Phyllanthaceae

Phyllanthaceae

Fabaceae

Primulaceae

Primulaceae
Abrus melanospermus Hassk.

Acampe praemorsa var. longepedunculata (Trimen) Govaerts

Acampe praemorsa (Roxb.) Blatt. \& Mccann

Achyrospermum wallichianum (Benth.) Benth. ex Hook.f.

Actinodaphne obovata (Nees) Blume

Aerides odorata Lour.

Aeschynanthus micranthus C.B.Clarke

Aglaia edulis (Roxb.) Wall.

Allophylus chartaceus (Kurz) Radlk.

Alpinia nigra (Gaertn.) Burtt

Alpinia roxburghii Sweet

Alseodaphne khasyana (Meisn.) Kosterm.

Alseodaphnopsis andersonii (King ex Hook.F.) H.W.Li \& J.Li

Amblyanthus glandulosus (Roxb.) A.DC.

Amorphophallus bulbifer (Roxb.) Blume

Amorphophallus napalensis (Wall.) Bogner \& Mayo

Antidesma acidum Retz.

Antidesma montanum Blume

Antidesma roxburghii Wall. ex Tul.

Aphanamixis polystachya (Wall.) R.Parker

Aporosa octandra (Buch.-Ham. ex D.Don) Vickery

Aporosa wallichii Hook.F.

Archidendron clypearia (Jack) I.C.Nielsen

Ardisia colorata G.Lodd.

Ardisia solanacea Roxb.

\section{Global IUCN
List status}

$\mathrm{NE}$

$\mathrm{NE}$

$\mathrm{NE}$

$\mathrm{NE}$

$\mathrm{NE}$

$\mathrm{NE}$

$\mathrm{NE}$

NT

$\mathrm{NE}$

NE

$\mathrm{NE}$

$\mathrm{NE}$

$\mathrm{NE}$

NE

$\mathrm{NE}$

$\mathrm{NE}$

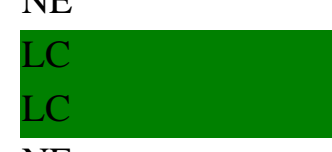

$\mathrm{NE}$

LC

$\mathrm{NE}$

LC
NE
NE
Habitat IUCN Growth

Form

Tree

Epiphyte

Epiphyte

Tree

Tree

Epiphyte

Epiphyte

Tree

BRF523 (G)

BRF801 (E)

BRF213 (H)

BRF564 (D)

BRF809 (B)

BRF242 (D)

BRF796 (G)

BRF514 (I)

BRF521 (B)

BRF371 (C)

BRF398 (I)

BRF123 (H)

BRF769 (B)

BRF126 (E)

BRF134 (A)

BRF353 (D)

BRF112 (B)

BRF370 (H)
Forb or Herb

Geophyte

Geophyte

Tree

Tree

Shrub

Geophyte

Geophyte

Shrub

Shrub

Shrub

Tree

Tree

Tree

Tree

Shrub

Shrub 
Aristolochiaceae

Aristolochiaceae

Aristolochiaceae

Moraceae

Menispermaceae

Malvaceae

Phyllanthaceae

Euphorbiaceae

Balanophoraceae

Apocynaceae

Begoniaceae

Lauraceae

Lauraceae

Lauraceae

Phyllanthaceae

Asteraceae

Gesneriaceae

Phyllanthaceae

Phyllanthaceae

Phyllanthaceae

Phyllanthaceae

Phyllanthaceae

Orchidaceae

Orchidaceae

Lamiaceae

Burseraceae

Burseraceae

Capparaceae
Aristolochia acuminata Lam.

Aristolochia assamica D.Borah \& T.V.Do

Aristolochia cathcartii Hook.F.

Artocarpus chama Buch.-Ham.

Aspidocarya uvifera Hook.F. \& Thomson

Ayenia grandifolia (DC.) Christenh. \& Byng

Baccaurea ramiflora Lour.

Balakata baccata (Roxb.) Esser

Balanophora dioica R.Br. ex Royle

Beaumontia grandiflora Wall.

Begonia silletensis (A.DC.) C.B.Clarke

Beilschmiedia assamica Meisn.

Beilschmiedia brandisii Hook.F.

Beilschmiedia roxburghiana Nees

Bischofia javanica Blume

Blumea balsamifera DC.

Boeica filiformis C.B.Clarke

Breynia androgyna (L.) Chakrab. \& N.P.Balakr.

Breynia retusa (Dennst.) Alston

Bridelia assamica Hook.F.

Bridelia retusa (L.) A.Juss.

Bridelia stipularis (L.) Blume

Bulbophyllum roxburghii (Lindl.) Rchb.F.

Calanthe masuca (D.Don) Lindl.

Callicarpa arborea Roxb.

Canarium bengalense Roxb.

Canarium resiniferum Bruce ex. King

Capparis multiflora Hook.F. \& Thomson

\begin{tabular}{|c|c|c|c|}
\hline $\mathrm{NE}$ & BRF201 (A) & PF & Vine \\
\hline $\mathrm{EN}^{*}$ & $\begin{array}{l}\text { BRF223 (D), } \\
\text { BRF978 (D) }\end{array}$ & SF & Vine \\
\hline $\mathrm{NE}$ & BRF515 (I) & PF & Vine \\
\hline $\mathrm{NE}$ & BRF524 (A) & $\mathrm{PF}, \mathrm{SF}$ & Tree \\
\hline $\mathrm{NE}$ & BRF238 (D) & $\mathrm{PF}$ & Vine \\
\hline $\mathrm{NE}$ & BRF780 (D) & PF, SF & Vine \\
\hline $\mathrm{LC}$ & BRF216 (H) & $\mathrm{PF}$ & Tree \\
\hline $\mathrm{LC}$ & BRF268 (C) & $\mathrm{PF}$ & Tree \\
\hline $\mathrm{NE}$ & BRF578 (B) & $\mathrm{PF}, \mathrm{SH}$ & Parasite \\
\hline $\mathrm{NE}$ & BRF513 (H) & SF & Vine \\
\hline $\mathrm{NE}$ & BRF248 (C) & $\mathrm{PF}$ & Forb or Herb \\
\hline $\mathrm{NE}$ & BRF230 (F) & $\mathrm{PF}$ & Tree \\
\hline $\mathrm{NE}$ & BRF574 (E) & $\mathrm{PF}$ & Tree \\
\hline $\mathrm{NE}$ & BRF375 (I) & PF & Tree \\
\hline $\mathrm{LC}$ & BRF115 (D) & $\mathrm{PF}, \mathrm{SF}$ & Tree \\
\hline LC & BRF250 (D) & $\mathrm{PF}$ & Forb or Herb \\
\hline $\mathrm{NE}$ & BRF547 (I) & $\mathrm{RH}$ & Shrub \\
\hline $\mathrm{NE}$ & BRF366 (C) & $\mathrm{PF}$ & Shrub \\
\hline $\mathrm{LC}$ & BRF397 (D) & $\mathrm{PF}$ & Shrub \\
\hline $\mathrm{NE}$ & BRF147 (B) & $\mathrm{PF}$ & Shrub \\
\hline $\mathrm{NE}$ & BRF387 (H) & $\mathrm{PF}$ & Tree \\
\hline $\mathrm{LC}$ & BRF793 (F) & SF & Shrub \\
\hline $\mathrm{NE}$ & BRF399 (G) & $\mathrm{PF}$ & Epiphyte \\
\hline $\mathrm{NE}$ & BRF568 (B) & $\mathrm{PF}$ & Geophyte \\
\hline $\mathrm{LC}$ & BRF356 (G) & $\mathrm{RF}$ & Tree \\
\hline $\mathrm{NE}$ & $\begin{array}{l}\text { BRF247 (D), } \\
\text { BRF974 (D) }\end{array}$ & $\mathrm{PF}$ & Tree \\
\hline $\mathrm{NE}$ & $\begin{array}{l}\text { BRF209 (G), } \\
\text { BRF979 (A) }\end{array}$ & PF & Tree \\
\hline $\mathrm{NE}$ & BRF263 (C) & $\mathrm{SH}$ & Shrub \\
\hline
\end{tabular}


Rhizophoraceae Carallia brachiata (Lour.) Merr.

Fagaceae

Rubiaceae

Rubiaceae

Meliaceae

Asparagaceae

Orchidaceae

Rutaceae

Rutaceae

Euphorbiaceae

Orchidaceae

Orchidaceae

Orchidaceae

Lamiaceae

Lamiaceae

Lamiaceae

Combretaceae

Commelinaceae

Boraginaceae

Orchidaceae

Capparaceae

Amaryllidaceae

Euphorbiaceae

Euphorbiaceae

Lauraceae

Hypoxidaceae

Orchidaceae

Orchidaceae

Fabaceae

Chrysoglossum ornatum Blume

Citrus indica Yu.Tanaka

Citrus medica L.

Claoxylon longipetiolatum Kurz

Cleisostoma subulatum Blume

Combretum acuminatum Roxb.

Connarus paniculatus Roxb.

Cordia myxa L.

Crateva religiosa G.Forst.

Croton caudatus Geiseler

Croton persimilis Müll.Arg.

Cryptocarya amygdalina Nees

Cymbidium aloifolium (L.) Sw.

Cymbidium bicolor Lindl.

Dalbergia rimosa Roxb.
Castanopsis lanceifolia (Oerst.) Hickel \& A.Camus

Catunaregam spinosa (Thunb.) Tirveng.

Chassalia curviflora (Wall.) Thwaites

Chisocheton cumingianus subsp. balansae (C.DC.) Mabb.

Chlorophytum assamicum D.Borah \& A.P.Da

Cleisocentron pallens (Cathcart ex Lindl.) N.Pearce \& P.J.Cribb

Cleisostoma tenuifolium (L.) Garay

Clerodendrum colebrookeanum Walp.

Clerodendrum bracteatum Wall. ex Walp.

Clerodendrum laevifolium Blume

Corymborkis veratrifolia (Reinw.) Blume

Crinum amoenum Ker Gawl. ex Roxb.

Curculigo capitulata (Lour.) Kuntze

\begin{tabular}{|c|c|c|c|}
\hline $\mathrm{NE}$ & BRF531 (E) & SF & Tree \\
\hline $\mathrm{NE}$ & BRF837 (F) & $\mathrm{PF}$ & Tree \\
\hline $\mathrm{NE}$ & BRF389 (D) & PF & Shrub \\
\hline $\mathrm{NE}$ & BRF536 (A) & $\mathrm{PF}$ & Shrub \\
\hline $\mathrm{LC}$ & BRF206 (A) & $\mathrm{PF}, \mathrm{RF}$ & Tree \\
\hline CR* & BRF262 (E) & $\mathrm{PF}$ & Forb or Herb \\
\hline $\mathrm{NE}$ & BRF527 (G) & $\mathrm{PF}$ & Forb or Herb \\
\hline $\mathrm{NE}$ & BRF34 (F) & $\mathrm{PF}$ & Shrub \\
\hline $\mathrm{NE}$ & BRF390 (I) & $\mathrm{RH}$ & Shrub \\
\hline $\mathrm{NE}$ & BRF805 (C) & SF & Forb or Herb \\
\hline $\mathrm{NE}$ & BRF383 (E) & $\mathrm{PF}$ & Epiphyte \\
\hline $\mathrm{NE}$ & BRF382 (D) & $\mathrm{PF}$ & Epiphyte \\
\hline $\mathrm{NE}$ & BRF789 (H) & $\mathrm{PF}$ & Epiphyte \\
\hline $\mathrm{NE}$ & BRF357 (E) & SF & Shrub \\
\hline $\mathrm{NE}$ & BRF559 (C) & $\mathrm{PF}$ & Shrub \\
\hline $\mathrm{NE}$ & BRF546 (D) & $\mathrm{PF}$ & Shrub \\
\hline $\mathrm{NE}$ & BRF517 (F) & PF & Vine \\
\hline $\mathrm{NE}$ & BRF836 (E) & $\mathrm{SH}$ & Vine \\
\hline $\mathrm{NE}$ & BRF573 (H) & SF & Tree \\
\hline $\mathrm{NE}$ & BRF575 (A) & $\mathrm{PF}$ & Geophyte \\
\hline $\mathrm{LC}$ & BRF766 (B) & $\mathrm{PF}, \mathrm{SF}$ & Tree \\
\hline $\mathrm{NE}$ & BRF226 (H) & RH & Geophyte \\
\hline $\mathrm{NE}$ & BRF577 (I) & SF & Vine \\
\hline $\mathrm{NE}$ & BRF267 (A) & $\mathrm{PF}$ & Tree \\
\hline $\mathrm{NE}$ & BRF520 (C) & $\mathrm{PF}$ & Tree \\
\hline $\mathrm{NE}$ & BRF354 (A) & $\mathrm{RH}$ & Forb or Herb \\
\hline $\mathrm{NE}$ & BRF219 (G) & $\mathrm{RF}$ & Epiphyte \\
\hline $\mathrm{NE}$ & BRF235 (I) & $\mathrm{PF}$ & Epiphyte \\
\hline $\mathrm{LC}$ & BRF548 (G) & $\mathrm{PF}$ & Tree \\
\hline
\end{tabular}


Staphyleaceae

Orchidaceae

Orchidaceae

Orchidaceae

Orchidaceae

Orchidaceae

Fabaceae

Acanthaceae

Orchidaceae

Dilleniaceae

Apocynaceae

Asparagaceae

Putranjivaceae

Lythraceae

Meliaceae

Meliaceae

Boraginaceae

Elaeagnaceae

Elaeocarpaceae

Elaeocarpaceae

Elaeocarpaceae

Araliaceae

Fabaceae

Orchidaceae

Olacaceae

Moraceae

Moraceae

Moraceae

Moraceae
Dalrympelea pomifera Roxb.

Dendrobium aphyllum (Roxb.) C.E.C.Fisch.

Dendrobium lituiflorum Lindl.

Dendrobium mannii Ridl.

Dendrobium moschatum (Banks) Sw.

Dendrolirium lasiopetalum (Willd.) S.C.Chen \& J.J.Wood

Derris scandens (Roxb.) Benth.

Dicliptera chinensis (L.) Juss.

Didymoplexis pallens Griff.

Dillenia indica L.

Dischidia bengalensis Colebr.

Dracaena petiolata Hook.f.

Drypetes assamica (Hook.F.) Pax \& K.Hoffm.

Duabanga grandiflora (Roxb. ex DC.) Walp.

Dysoxylum excelsum Blume

Dysoxylum gotadhora (Buch.-Ham.) Mabb.

Ehretia wallichiana Hook.f. \& Thomson ex C.B.Clarke

Elaeagnus pyriformis Hook.f.

Elaeocarpus angustifolius Blume

Elaeocarpus rugosus Roxb. ex G.Don

Elaeocarpus varunua Buch.-Ham. ex Mast.

Eleutherococcus trifoliatus (L.) S.Y.Hu var. trifoliatus

Entada phaseoloides (L.) Merr.

Epipogium roseum (D.Don) Lindl.

Erythropalum scandens Blume

Ficus auriculata Lour.

Ficus benjamina L.

Ficus drupacea Thunb.

Ficus elastica Roxb. ex Hornem.

\begin{tabular}{|c|c|c|c|}
\hline LC & BRF391 (C) & $\mathrm{PF}, \mathrm{SF}$ & Tree \\
\hline LC & BRF101 (G) & $\mathrm{RF}$ & Epiphyte \\
\hline NE & BRF538 (H) & $\mathrm{RF}$ & Epiphyte \\
\hline $\mathrm{NE}$ & BRF102 (B) & PF & Epiphyte \\
\hline $\mathrm{NE}$ & BRF791 (G) & PF & Epiphyte \\
\hline $\mathrm{NE}$ & BRF364 (F) & PF & Epiphyte \\
\hline $\mathrm{LC}$ & BRF353 (A) & RH & Vine \\
\hline $\mathrm{NE}$ & BRF261 (A) & SF & Forb or Herb \\
\hline NE & BRF137 (D) & SH & Geophyte \\
\hline NE & BRF264 (D) & \multicolumn{2}{|c|}{ PF, SF, RH Tree } \\
\hline NE & BRF579 (F) & $\mathrm{PF}, \mathrm{SF}$ & Epiphyte \\
\hline NE & BRF814 (G) & $\mathrm{PF}$ & Forb or Herb \\
\hline NE & BRF566 (I) & PF & Tree \\
\hline $\mathrm{LC}$ & BRF544 (B) & $\mathrm{RF}$ & Tree \\
\hline $\mathrm{NE}$ & BRF773 (I) & $\mathrm{PF}$ & Tree \\
\hline $\mathrm{NE}$ & BRF775 (E) & PF & Tree \\
\hline NE & BRF822 (E) & PF & Shrub \\
\hline $\mathrm{NE}$ & BRF827 (G) & RH & Shrub \\
\hline $\mathrm{LC}$ & BRF227 (G) & $\mathrm{PF}$ & Tree \\
\hline VU & BRF207 (C) & PF & Tree \\
\hline NE & $\begin{array}{l}\text { BRF228 (F), } \\
\text { BRF976 (D) }\end{array}$ & PF & Tree \\
\hline $\mathrm{NE}$ & BRF804 (C) & RH & Shrub \\
\hline NE & BRF229 (C) & PF & Vine \\
\hline NE & BRF837 (F) & PF & Geophyte \\
\hline $\mathrm{LC}$ & BRF381 (B) & SF & Vine \\
\hline LC & BRF378 (G) & $\mathrm{PF}, \mathrm{SF}$ & Tree \\
\hline $\mathrm{LC}$ & BRF569 (B) & $\mathrm{PF}, \mathrm{SF}$ & Tree \\
\hline LC & BRF233 (I) & $\mathrm{PF}, \mathrm{SF}$ & Tree \\
\hline NE & BRF774 (B) & $\mathrm{PF}, \mathrm{SF}$ & Tree \\
\hline
\end{tabular}




\begin{tabular}{|c|c|c|c|c|c|}
\hline Moraceae & Ficus hederacea Roxb. & $\mathrm{NE}$ & BRF237 (E) & $\mathrm{PF}, \mathrm{SF}$ & Vine \\
\hline Moraceae & Ficus heterophylla L.F. & $\mathrm{NE}$ & BRF541 (C) & $\mathrm{RH}$ & Vine \\
\hline Moraceae & Ficus heteropleura Blume & $\mathrm{NE}$ & BRF363 (E) & $\mathrm{PF}, \mathrm{SF}$ & Vine \\
\hline Moraceae & Ficus hispida L.F. & $\mathrm{LC}$ & BRF108 (H) & $\mathrm{SF}$ & Tree \\
\hline Moraceae & Ficus nervosa B.Heyne ex Roth & $\mathrm{LC}$ & BRF119 (A) & $\mathrm{PF}, \mathrm{SF}$ & Tree \\
\hline Moraceae & Ficus semicordata Buch.-Ham. ex Sm. & $\mathrm{LC}$ & BRF141 (D) & $\mathrm{RH}$ & Tree \\
\hline Moraceae & Ficus subincisa Buch.Ham. ex Sm. & $\mathrm{LC}$ & BRF407 (B) & $\mathrm{PF}$ & Vine \\
\hline Moraceae & Ficus subulata Blume & $\mathrm{NE}$ & BRF205 (B) & PF & Vine \\
\hline Moraceae & Ficus tinctoria G.Forst. & $\mathrm{LC}$ & BRF151 (I) & $\mathrm{PF}$ & Vine \\
\hline Annonaceae & Fissistigma bicolor (Roxb.) Merr. & $\mathrm{NE}$ & BRF258 (C) & SF & Vine \\
\hline Annonaceae & Fissistigma polyanthum (Hook.f. \& Thomson) Merr. & $\mathrm{NE}$ & BRF225 (H) & SF & Vine \\
\hline Annonaceae & Friesodielsia fornicata (Roxb.) D.Das & NE & $\begin{array}{l}\text { BRF767 (C), } \\
\text { BRF977 (D) }\end{array}$ & $\mathrm{PF}$ & Vine \\
\hline Clusiaceae & Garcinia xanthochymus Hook.F. ex T.Anderson & NE & BRF580 (F) & $\mathrm{PF}$ & Tree \\
\hline Orchidaceae & Gastrochilus obliquus var. suavis (Seidenf.) Z.H.Tsi & $\mathrm{NE}$ & BRF528 (C) & $\mathrm{PF}$ & Epiphyte \\
\hline Zingiberaceae & Globba multiflora Wall. ex Baker & $\mathrm{NE}$ & BRF534 (B) & GR & Geophyte \\
\hline Phyllanthaceae & $\begin{array}{l}\text { Glochidion zeylanicum var. arborescens (Blume) Chakrab. \& } \\
\text { M.Gangop. }\end{array}$ & $\mathrm{NE}$ & BRF794 (E) & SF & Tree \\
\hline Lamiaceae & Gomphostemma niveum Hook.F. & $\mathrm{NE}$ & BRF358 (F) & $\mathrm{PF}$ & Forb or Herb \\
\hline Annonaceae & Goniothalamus simonsii Hook.f. \& Thomson & $\mathrm{LC}$ & BRF784 (E) & $\mathrm{PF}$ & Shrub \\
\hline Rhamnaceae & Gouania leptostachya DC. & $\mathrm{NE}$ & BRF121 (F) & SF & Vine \\
\hline Malvaceae & Grewia serrulata DC. & $\mathrm{NE}$ & BRF558 (E) & SF & Shrub \\
\hline Achariaceae & Gynocardia odorata R.Br. & $\mathrm{NE}$ & BRF512 (F) & $\mathrm{PF}$ & Tree \\
\hline Zingiberaceae & Hedychium coccineum Buch.-Ham. ex Sm. & NE & BRF212 (C) & SF, SH & Geophyte \\
\hline Zingiberaceae & Hedychium stenopetalum G.Lodd. & $\mathrm{NE}$ & BRF837 (F) & $\mathrm{OF}$ & Geophyte \\
\hline Loranthaceae & Helixanthera ligustrina (Wall.) Danser & NE & BRF106 (C) & $\mathrm{PF}$ & Parasite \\
\hline Orchidaceae & Hetaeria affinis (Griff.) Seidenf. \& Ormerod & $\mathrm{NE}$ & BRF384 (I) & $\mathrm{PF}$ & Geophyte \\
\hline Apocynaceae & Heterostemma alatum Wight \&Arn. & NE & BRF202 (G) & $\mathrm{PF}$ & Vine \\
\hline Malvaceae & Hibiscus fragrans Roxb. & $\mathrm{NE}$ & BRF779 (B) & $\mathrm{PF}$ & Vine \\
\hline Malpighiaceae & Hiptage benghalensis (L.) Kurz & LC & BRF361 (H) & $\mathrm{PF}$ & Vine \\
\hline
\end{tabular}




\begin{tabular}{|c|c|c|c|c|c|}
\hline Cucurbitaceae & Hodgsonia macrocarpa (Blume) Cogn. & NE & $\begin{array}{l}\text { BRF208 (C), } \\
\text { BRF975 (D) }\end{array}$ & $\mathrm{PF}$ & Vine \\
\hline Araceae & Homalomena aromatica (Spreng.) Schott & $\mathrm{NE}$ & $\mathrm{BRF} 210(\mathrm{G})$ & SF & Geophyte \\
\hline Myristicaceae & Horsfieldia kingii (Hook.f.) Warb. & $\mathrm{NE}$ & BRF781 (C) & $\mathrm{PF}$ & Tree \\
\hline Apocynaceae & Hoya verticillata (Vahl) G.Don & $\mathrm{NE}$ & BRF254 (D) & $\mathrm{PF}, \mathrm{SF}$ & Epiphyte \\
\hline Menispermaceae & Hypserpa nitida Miers ex Benth. & $\mathrm{NE}$ & BRF542 (H) & $\mathrm{PF}$ & Vine \\
\hline Rubiaceae & Ixora nigricans R.Br. ex Wight \& Arn. & $\mathrm{NE}$ & BRF395 (G) & $\mathrm{PF}$ & Shrub \\
\hline Oleaceae & Jasminum coarctatum Roxb. & $\mathrm{NE}$ & BRF400 (I) & SF, SH & Vine \\
\hline Oleaceae & Jasminum laurifolium Roxb. ex Hornem. & $\mathrm{NE}$ & BRF234 (D) & $\mathrm{SF}, \mathrm{SH}$ & Vine \\
\hline Myristicaceae & Knema angustifolia (Roxb.) Warb. & $\mathrm{NE}$ & BRF450 (I) & $\mathrm{PF}$ & Tree \\
\hline Malvaceae & Kydia calycina Roxb. & $\mathrm{LC}$ & BRF373 (A) & $\mathrm{RF}$ & Tree \\
\hline Zingiberaceae & Larsenianthus careyanus (Benth. \& Hook.F.) W.J.Kress \& Mood & $\mathrm{NE}$ & BRF554 (E) & SF, SH & Geophyte \\
\hline Araceae & Lasia spinosa (L.) Thwaites & $\mathrm{LC}$ & BRF252 (D) & RH & Forb or Herb \\
\hline Vitaceae & Leea compactiflora Kurz & $\mathrm{NE}$ & BRF392 (A) & SF & Geophyte \\
\hline Opiliaceae & Lepionurus sylvestris Blume & $\mathrm{NE}$ & BRF539 (B) & $\mathrm{PF}$ & Shrub \\
\hline Sapindaceae & Lepisanthes senegalensis (Poir.) Leenh. & $\mathrm{NE}$ & BRF535 (H) & $\mathrm{PF}$ & Shrub \\
\hline Lauraceae & Lindera reticulata (Blume) Benth. \& Hook.F. ex Fern.-Vill. & $\mathrm{NE}$ & BRF571 (G) & $\mathrm{PF}$ & Tree \\
\hline Altingiaceae & Liquidambar excelsa (Noronha) Oken & $\mathrm{NE}$ & BRF211 (E) & $\mathrm{PF}$ & Tree \\
\hline Fagaceae & Lithocarpus listeri (King) Grierson \& D.G.Long & $\mathrm{NE}$ & BRF835 (B) & $\mathrm{PF}$ & Tree \\
\hline Lauraceae & Litsea albescens (Hook.f.) D.G.Long & $\mathrm{NE}$ & BRF768 (I) & $\mathrm{PF}$ & Tree \\
\hline Lauraceae & Litsea assamica Hook.F. & $\mathrm{NE}$ & BRF360 (F) & $\mathrm{PF}$ & Tree \\
\hline Lauraceae & Litsea chartacea Hook.F. & $\mathrm{NE}$ & BRF103 (A) & $\mathrm{PF}$ & Tree \\
\hline Lauraceae & Litsea glutinosa (Lour.) C.B.Rob. & $\mathrm{LC}$ & BRF104 (I) & $\mathrm{PF}$ & Tree \\
\hline Lauraceae & Litsea hookeri (Meisn.) D.G.Long & $\mathrm{NE}$ & BRF545 (G) & RH & Shrub \\
\hline Lauraceae & Litsea khasyana Meisn. & $\mathrm{NE}$ & BRF576 (D) & $\mathrm{RH}, \mathrm{RH}$ & Shrub \\
\hline Lauraceae & Litsea laeta (Nees) Trimen & $\mathrm{LC}$ & BRF770 (E) & $\mathrm{PF}$ & Tree \\
\hline Lauraceae & Litsea monopetala (Roxb.) Pers. & $\mathrm{NE}$ & BRF241 (I) & $\mathrm{PF}, \mathrm{SF}$ & Tree \\
\hline Lauraceae & Litsea sericea (Wall. ex Nees) Hook.F. & LC & BRF105 (A) & $\mathrm{PF}$ & Tree \\
\hline Campanulaceae & Lobelia alsinoides Lam. & LC & BRF246 (I) & $\mathrm{RH}, \mathrm{SF}$ & Forb or Herb \\
\hline Campanulaceae & Lobelia zeylanica L. & LC & BRF516 (E) & $\mathrm{RH}, \mathrm{SF}$ & Forb or Herb \\
\hline
\end{tabular}


Orchidaceae

Solanaceae

Lauraceae

Lauraceae

Moraceae

Araliaceae

Loranthaceae

Primulaceae

Magnoliaceae

Magnoliaceae

Magnoliaceae

Euphorbiaceae

Euphorbiaceae

Melastomataceae

Sabiaceae

Sabiaceae

Calophyllaceae

Fabaceae

Fabaceae

Malvaceae

Rutaceae

Annonaceae

Icacinaceae

Annonaceae

Moraceae

Moraceae

Commelinaceae

Musaceae
Luisia trichorrhiza (Hook.) Blume

Lycianthes biflora (Lour.) Bitter

Machilus duthiei King ex Hook.f.

Machilus gamblei King ex Hook.F.

Maclura cochinchinensis (Lour.) Corner

Macropanax dispermus (Blume) Kuntze

Macrosolen cochinchinensis (Lour.) Tiegh.

Maesa indica (Roxb.) Sweet

Magnolia griffithii Hook.F. \& Thomson

Magnolia hodgsonii (Hook.F. \& Thomson) H.Keng

Magnolia kingii (Dandy) Figlar

Mallotus philippensis (Lam.) Müll.Arg.

Mallotus roxburghianus Müll.Arg.

Melastoma malabathricum L.

Meliosma pinnata (Roxb.) Maxim

Meliosma simplicifolia (Roxb.) Walp.

Mesua ferrea L.

Mezoneuron cucullatum (Roxb.) Wight \& Arn.

Mezoneuron enneaphyllum (Roxb.) Wight \& Arn. ex Voigt

Microcos paniculata L.

Micromelum integerrimum (Roxb. ex DC.) Wight \& Arn. ex

M.Roem.

Miliusa dioeca (Roxb.) Chaowasku \& Kessler

Miquelia assamica (Griff.) Mast. ex B.D.Jacks.

Mitrephora tomentosa Hook.F. \& Thomson

Morus alba L.

Morus macroura Miq.

Murdannia japonica (Thunb.) Faden

Musa velutina H.Wendl. \& Drude

\begin{tabular}{|c|c|c|c|}
\hline $\mathrm{NE}$ & BRF385 (H) & PF & Epiphyte \\
\hline $\mathrm{NE}$ & BRF214 (A) & SF & Shrub \\
\hline $\mathrm{NE}$ & BRF771 (D) & $\mathrm{PF}$ & Tree \\
\hline $\mathrm{LC}$ & BRF401 (E) & $\mathrm{PF}$ & Tree \\
\hline $\mathrm{NE}$ & BRF525 (E) & SF & Shrub \\
\hline $\mathrm{LC}$ & BRF826 (I) & PF & Tree \\
\hline $\mathrm{NE}$ & BRF231 (A) & $\mathrm{PF}$ & Parasite \\
\hline $\mathrm{LC}$ & BRF110 (C) & SF & Shrub \\
\hline $\mathrm{DD}$ & $\operatorname{BRF} 376(\mathrm{G})$ & PF & Tree \\
\hline $\mathrm{LC}$ & BRF240 (E) & PF, SF & Tree \\
\hline DD & BRF777 (D) & $\mathrm{PF}$ & Tree \\
\hline $\mathrm{LC}$ & BRF269 (G) & SF & Tree \\
\hline $\mathrm{NE}$ & BRF351 (D) & SF & Shrub \\
\hline $\mathrm{NE}$ & BRF107 (A) & SF & Shrub \\
\hline $\mathrm{NE}$ & BRF797 (G) & $\mathrm{PF}$ & Tree \\
\hline $\mathrm{NE}$ & BRF565 (D) & SF & Tree \\
\hline $\mathrm{NE}$ & BRF550 (F) & $\mathrm{PF}$ & Tree \\
\hline $\mathrm{NE}$ & BRF831 (I) & SH & Vine \\
\hline $\mathrm{NE}$ & BRF244 (A) & SH & Vine \\
\hline $\mathrm{LC}$ & BRF776 (I) & $\mathrm{PF}$ & Tree \\
\hline $\mathrm{LC}$ & BRF532 (B) & SF & Tree \\
\hline $\mathrm{NE}$ & $\begin{array}{l}\text { BRF257 (F), } \\
\text { BRF973 (D) }\end{array}$ & $\mathrm{PF}$ & Tree \\
\hline $\mathrm{NE}$ & BRF355 (I) & $\mathrm{PF}$ & Vine \\
\hline $\mathrm{NE}$ & BRF572 (B) & PF & Tree \\
\hline $\mathrm{NE}$ & BRF782 (G) & $\mathrm{SF}$ & Shrub \\
\hline $\mathrm{NE}$ & BRF783 (E) & $\mathrm{PF}$ & Tree \\
\hline $\mathrm{NE}$ & BRF832 (G) & $\mathrm{SF}$ & Forb or $\mathrm{H}$ \\
\hline $\mathrm{NE}$ & BRF379 (D) & GR & Geophyte \\
\hline
\end{tabular}


Rubiaceae

Rubiaceae

Rubiaceae

Nyssaceae

Apiaceae

Asparagaceae

Rubiaceae

Urticaceae

Melastomataceae

Pandanaceae

Orchidaceae

Menispermaceae

Rubiaceae

Anacardiaceae

Asparagaceae

Orchidaceae

Orchidaceae

Orchidaceae

Lauraceae

Orchidaceae

Marantaceae

Phyllanthaceae

Simaroubaceae

Orchidaceae

Piperaceae

Piperaceae

Urticaceae

Commelinaceae

Annonaceae
Mussaenda glabra Vahl

Mussaenda roxburghii Hook.F.

Mycetia nutans (R.Br. ex Kurz) Razafim. \& B.Bremer

Nyssa javanica (Blume) Wangerin

Oenanthe javanica (Blume) DC.

Ophiopogon micranthus Hook.f.

Ophiorrhiza ochroleuca Hook.F.

Oreocnide integrifolia (Gaudich.) Miq.

Osbeckia nepalensis Hook.F.

Pandanus unguifer Hook.f.

Papilionanthe teres (Roxb.) Schltr.

Parabaena sagittata Miers ex Hook.F. \& Thomson

Pavetta indica L.

Pegia nitida Colebr.

Peliosanthes macrophylla var. assamensis N.Tanaka \& D.Borah

Phalaenopsis deliciosa Rchb.F.

Phalaenopsis lobbii (Rchb.F.) H.R.Sweet

Phalaenopsis mannii Rchb.F.

Phoebe attenuata (Nees) Nees

Pholidota imbricata Hook.

Phrynium pubinerve Blume

Phyllanthus assamicus Müll.Arg.

Picrasma javanica Blume

Pinalia bractescens (Lindl.) Kuntze

Piper betleoides C.DC.

Piper sylvaticum Roxb.

Poikilospermum suaveolens (Blume) Merr.

Pollia subumbellata C.B.Clarke

Polyalthia suberosa (Roxb.) Thwaites
$\mathrm{NE}$

$\mathrm{NE}$

$\mathrm{NE}$

NE

$\mathrm{NE}$

NE

$\mathrm{NE}$

NE

$\mathrm{NE}$

NE

$\mathrm{NE}$

NE

$\mathrm{NE}$

$\mathrm{NE}$

$\mathrm{NE}$

NE

NE

NE

NE

$\mathrm{NE}$

NE

LC

NE

$\mathrm{NE}$

$\mathrm{NE}$

$\mathrm{NE}$

NE

NE

\begin{tabular}{|c|c|c|}
\hline BRF396 (D) & PF & Shrub \\
\hline BRF369 (C) & SF & Shrub \\
\hline BRF128 (I) & $\mathrm{PF}$ & Shrub \\
\hline BRF380 (I) & $\mathrm{PF}$ & Tree \\
\hline BRF255 (D) & $\mathrm{RH}$ & Forb or Herb \\
\hline BRF818 (F) & PF, SH & Forb or Herb \\
\hline BRF114 (E) & $\mathrm{PF}$ & Shrub \\
\hline BRF368 (C) & $\begin{array}{l}\mathrm{PF}, \mathrm{RH}, \\
\mathrm{RH}\end{array}$ & Tree \\
\hline BRF362 (I) & SF & Shrub \\
\hline BRF785 (E) & RH & Shrub \\
\hline BRF567 (F) & RF & Epiphyte \\
\hline BRF221 (B) & $\mathrm{PF}, \mathrm{SF}$ & Vine \\
\hline BRF132 (H) & PF & Shrub \\
\hline BRF259 (I) & SF & Vine \\
\hline BRF837 (F) & PF & Forb or Herb \\
\hline BRF217 (C) & $\mathrm{PF}$ & Epiphyte \\
\hline BRF372 (G) & PF & Epiphyte \\
\hline BRF131 (E) & PF & Epiphyte \\
\hline BRF521 (C) & $\mathrm{PF}$ & Tree \\
\hline BRF418 (I) & $\mathrm{PF}$ & Epiphyte \\
\hline BRF232 (B) & RH & Forb or Herb \\
\hline BRF149 (G) & $\mathrm{PF}$ & Tree \\
\hline BRF803 (A) & $\mathrm{PF}$ & Tree \\
\hline BRF787 (A) & $\mathrm{PF}$ & Epiphyte \\
\hline BRF411 (D) & $\mathrm{PF}, \mathrm{SF}$ & Vine \\
\hline BRF530 (I) & $\mathrm{PF}, \mathrm{SF}$ & Vine \\
\hline BRF795 (D) & $\mathrm{PF}, \mathrm{SF}$ & Vine \\
\hline BRF560 (B) & $\mathrm{PF}$ & Forb or Herb \\
\hline BRF256 (G) & $\mathrm{PF}$ & Shrub \\
\hline
\end{tabular}




\begin{tabular}{|c|c|c|c|c|c|}
\hline Orchidaceae & Pomatocalpa spicatum Breda & $\mathrm{NE}$ & BRF386 (A) & $\mathrm{PF}$ & Epiphyte \\
\hline Orchidaceae & Pomatocalpa undulatum (Lindl.) J.J.Sm. & $\mathrm{NE}$ & BRF145 (A) & PF & Epiphyte \\
\hline Urticaceae & Pouzolzia sanguinea (Blume) Merr. & $\mathrm{NE}$ & BRF799 (F) & SF & Vine \\
\hline Lamiaceae & Premna bengalensis C.B.Clarke & $\mathrm{NE}$ & BRF243 (I) & $\mathrm{PF}$ & Tree \\
\hline Lamiaceae & Premna esculenta Roxb. & $\mathrm{NE}$ & BRF359 (A) & $\mathrm{PF}$ & Tree \\
\hline Burseraceae & Protium serratum (Wall. ex Colebr.) Engl. & $\mathrm{NE}$ & BRF829 (I) & $\mathrm{PF}$ & Tree \\
\hline Acanthaceae & Pseuderanthemum latifolium (Vahl) B.Hansen & $\mathrm{NE}$ & BRF553 (F) & SF & Forb or Herb \\
\hline Poaceae & Pseudostachyum polymorphum Munro & $\mathrm{NE}$ & BRF798 (D) & GR & Forb or Herb \\
\hline Rubiaceae & Psychotria denticulata Wall. & $\mathrm{NE}$ & BRF837 (F) & $\mathrm{PF}$ & Forb or Herb \\
\hline Malvaceae & Pterospermum acerifolium (L.) Willd. & $\mathrm{NE}$ & BRF374 (B) & $\mathrm{PF}$ & Tree \\
\hline Malvaceae & Pterospermum lanceifolium Roxb. ex DC. & $\mathrm{NE}$ & BRF239 (I) & $\mathrm{PF}$ & Tree \\
\hline Menispermaceae & Pycnarrhena pleniflora Miers ex Hook.F. \& Thomson & $\mathrm{NE}$ & BRF204 (H) & $\mathrm{PF}$ & Vine \\
\hline Theaceae & Pyrenaria khasiana var. lakhimpurense N. Odyuo \& D.K. Roy & $\mathrm{NE}$ & BRF393 (F) & $\mathrm{PF}$ & Tree \\
\hline Rhamnaceae & Rhamnus napalensis (Wall.) M.A.Lawson & $\mathrm{LC}$ & BRF215 (B) & GR & Tree \\
\hline Orchidaceae & Rhynchostylis retusa (L.) Blume & $\mathrm{NE}$ & BRF537 (F) & RF & Epiphyte \\
\hline Rosaceae & Rubus moluccanus L. & $\mathrm{NE}$ & BRF800 (G) & SF & Shrub \\
\hline Sabiaceae & Sabia lanceolata Colebr. & $\mathrm{NE}$ & BRF555 (C) & $\mathrm{PF}$ & Vine \\
\hline Actinidiaceae & Saurauia armata Kurz & $\mathrm{NE}$ & BRF260 (C) & $\mathrm{PF}$ & Tree \\
\hline Actinidiaceae & Saurauia napaulensis DC. & $\mathrm{LC}$ & BRF563 (B) & SF & Tree \\
\hline Marantaceae & Schumannianthus benthamianus (Kuntze) Veldkamp \& I.M.Turner & $\mathrm{NE}$ & BRF543 (I) & GR & Forb or Herb \\
\hline Elaeocarpaceae & Sloanea sterculiacea var. assamica (Benth.) Coode & $\mathrm{NE}$ & BRF266 (E) & $\mathrm{PF}$ & Tree \\
\hline Smilacaceae & Smilax orthoptera A.DC. & NE & BRF367 (D) & $\mathrm{SH}$ & Vine \\
\hline Rubiaceae & Spiradiclis bifida Kurz & $\mathrm{NE}$ & BRF790 (F) & $\mathrm{PF}$ & Forb or Herb \\
\hline Stachyuraceae & Stachyurus himalaicus Hook.f. \& Thomson ex Benth. & $\mathrm{LC}$ & BRF786 (I) & $\mathrm{PF}$ & Shrub \\
\hline Gesneriaceae & Stauranthera umbrosa (Griff.) C.B.Clarke & $\mathrm{NE}$ & BRF810 (C) & $\mathrm{RH}$ & Forb or Herb \\
\hline Stemonaceae & Stemona tuberosa Lour. & $\mathrm{NE}$ & BRF533 (H) & SF & Vine \\
\hline Menispermaceae & Stephania rotunda Lour. & NE & BRF377 (I) & SF & Vine \\
\hline Malvaceae & Sterculia lanceolata var. coccinea (Jack) Phengklai & $\mathrm{NE}$ & BRF522 (C) & $\mathrm{PF}$ & Shrub \\
\hline Malvaceae & Sterculia villosa Roxb. ex Sm. & NE & BRF570 (E) & RF & Tree \\
\hline
\end{tabular}


Resedaceae

Acanthaceae

Apocynaceae

Styracaceae

Styracaceae

Myrtaceae

Myrtaceae

Myrtaceae

Combretaceae

Combretaceae

Dilleniaceae

Loranthaceae

Orchidaceae

Orchidaceae

Asparagaceae

Apocynaceae

Apocynaceae

Lamiaceae

Lamiaceae

Lamiaceae

Campanulaceae

Apocynaceae

Cucurbitaceae

Rutaceae

Rutaceae

Orchidaceae

Rhamnaceae
Stixis suaveolens (Roxb.) Baill.

Strobilanthes paniculiformis J.R.I.Wood

Strophanthus wallichii A.DC.

Styrax hookeri C.B.Clarke

Styrax serrulatus Roxb.

Syzygium aqueum (Burm.F.) Alston

Syzygium balsameum (Wight) Wall. ex Walp.

Syzygium oblatum (Roxb.) Wall. ex A.M.Cowan \& Cowan

Terminalia bellirica (Gaertn.) Roxb.

Terminalia citrina (Gaertn.) Roxb.

Tolypanthus maclurei (Merr.) Danser

Tropidia angulosa (Lindl.) Blume

Tropidia curculigoides Lindl.

Tupistra stoliczana Kurz

Urceola rosea (Hook. \& Arn.) D.J. Middleton

Vallaris solanacea (Roth) Kuntze

Vitex glabrata R.Br.

Vitex pinnata $\mathrm{L}$.

Vitex quinata (Lour.) F.N.Williams

Wahlenbergia marginata (Thunb.) A.DC.

Wrightia arborea (Dennst.) Mabb.

Zanonia indica $\mathrm{L}$.

Zanthoxylum oxyphyllum Edgew.

Zanthoxylum rhetsa (Roxb.) DC.

Zeuxine nervosa (Wall. ex Lindl.) Benth. ex Trimen

Ziziphus funiculosa Buch.-Ham. ex M.A.Lawson
Tetracera sarmentosa (L.) Vahl

\begin{tabular}{|c|c|c|c|}
\hline NE & BRF388 (A) & $\mathrm{PF}, \mathrm{SF}$ & Vine \\
\hline $\mathrm{NE}$ & BRF766 (G) & $\mathrm{RH}$ & Forb or Herb \\
\hline NE & BRF224 (C) & $\mathrm{PF}$ & Vine \\
\hline NE & BRF788 (E) & $\mathrm{PF}$ & Tree \\
\hline $\mathrm{NE}$ & BRF125 (B) & RH & Tree \\
\hline NE & BRF220 (B) & $\mathrm{PF}$ & Tree \\
\hline NE & BRF236 (E) & $\mathrm{PF}, \mathrm{SF}$ & Tree \\
\hline NE & BRF772 (A) & $\mathrm{PF}$ & Tree \\
\hline NE & BRF549 (D) & PF & Tree \\
\hline $\mathrm{LC}$ & BRF834 (I) & $\mathrm{PF}$ & Tree \\
\hline NE & BRF265 (B) & $\mathrm{PF}, \mathrm{SF}$ & Vine \\
\hline NE & BRF203 (H) & $\mathrm{PF}, \mathrm{SF}$ & Parasite \\
\hline NE & BRF778 (H) & PF & Forb or Herb \\
\hline NE & BRF529 (B) & PF & Forb or Herb \\
\hline NE & BRF249 (C) & PF & Forb or Herb \\
\hline NE & BRF552 (I) & $\mathrm{PF}$ & Vine \\
\hline NE & BRF253 (E) & PF & Vine \\
\hline $\mathrm{LC}$ & BRF830 (F) & PF & Tree \\
\hline LC & BRF807 (I) & PF & Tree \\
\hline $\mathrm{LC}$ & BRF808 (B) & PF & Tree \\
\hline $\mathrm{NE}$ & BRF245 (H) & $\mathrm{RH}, \mathrm{SF}$ & Forb or Herb \\
\hline NE & BRF562 (H) & $\mathrm{PF}$ & Tree \\
\hline NE & BRF222 (A) & SF & Vine \\
\hline NE & BRF802 (D) & PF & Vine \\
\hline $\mathrm{LC}$ & BRF792 (I) & PF & Tree \\
\hline NE & BRF365 (D) & PF & Geophyte \\
\hline NE & BRF556 (G) & PF & Vine \\
\hline
\end{tabular}
Shrublands, RH - Riparian habitats, GR - Grasslands. 


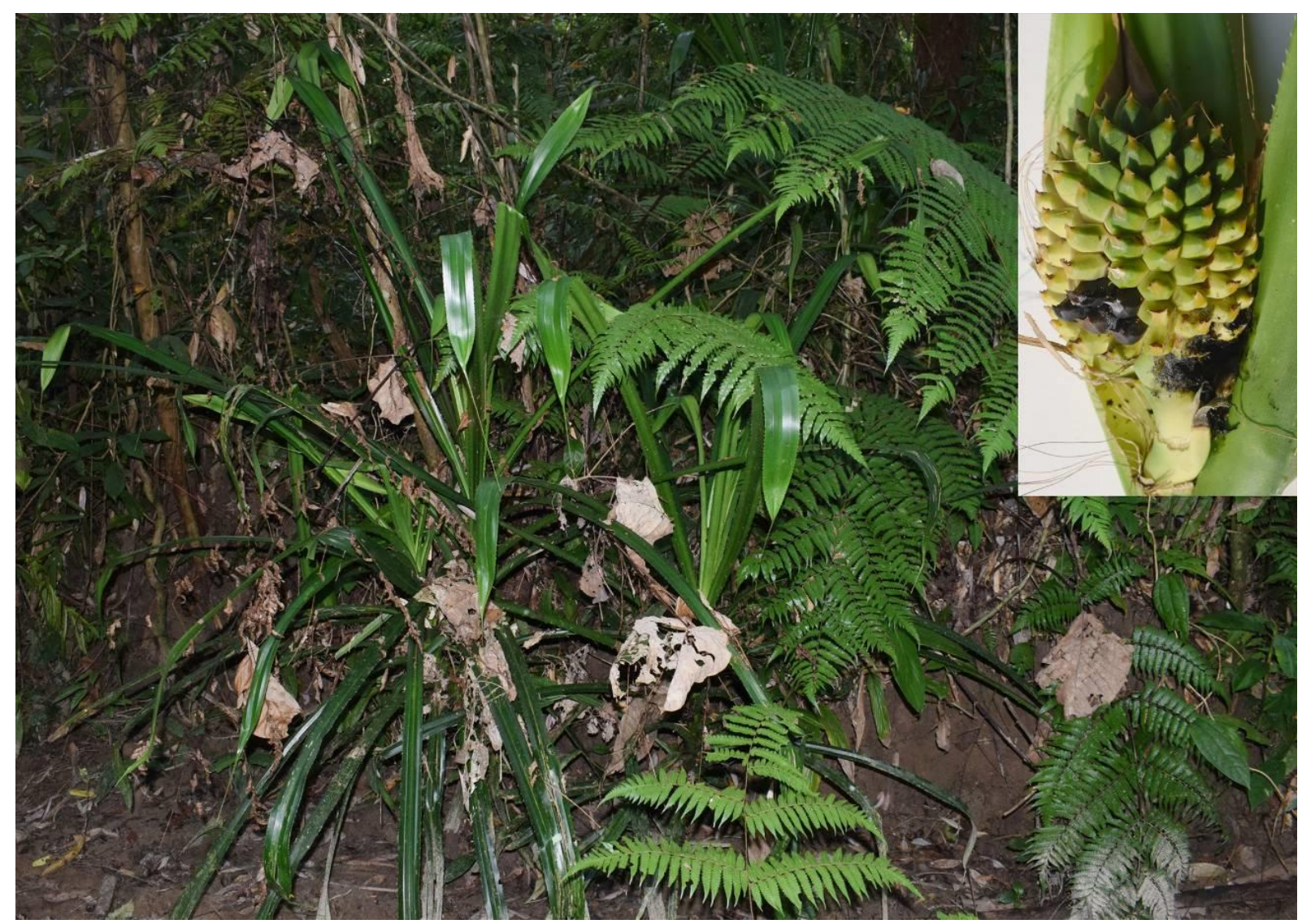

Fig. 2: Pandanus unguifer in Behali Reserve Forest (India); inset: syncarp.

The families with highest species number were Orchidaceae (34 species), Lauraceae (20), Moraceae (17), Phyllanthaceae (14) and Lamiaceae (11 species). The richest genera by number of species were Ficus and Litsea with 13 and 9 species, respectively.

Analysis of vascular plant flora in terms of growth forms demonstrated the predominance of trees (37.0\%), followed by vines (18.1\%) and shrubs (16.4\%), and so on (Fig. 3).

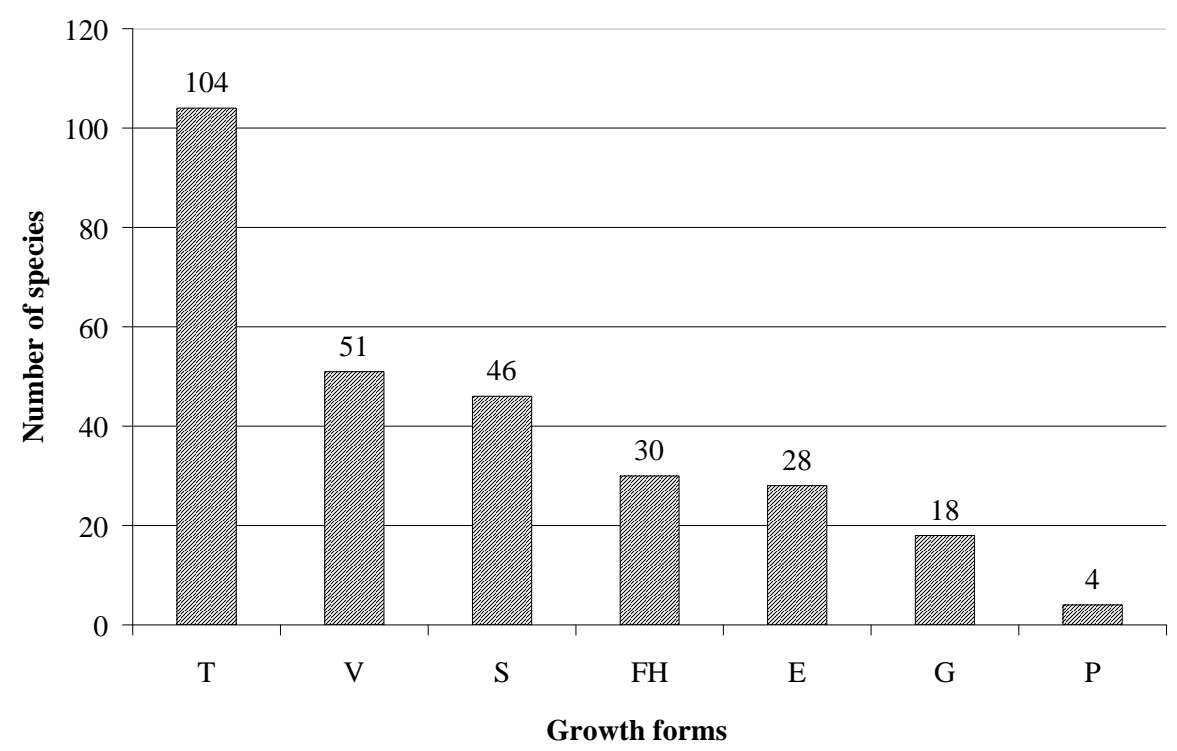

Fig. 3: Distribution of vascular plant species along growth forms. Designations: $\mathrm{T}-$ tree, $\mathrm{V}-\mathrm{vine}, \mathrm{S}-\mathrm{shrub}, \mathrm{E}$ - epiphyte, FH - forb or herb, G - geophyte, $\mathrm{P}$ - parasite. 
In the present study, we estimated the habitat diversity where the vascular plants grow in the BRF (Fig. 4). We found that the highest number of species was registered in pristine forests (190 taxa) followed by secondary forests (76 taxa) and rehabilitated forests (26 taxa). At the same time, open habitats (e.g. grasslands) were characterized by the lowest species richness.

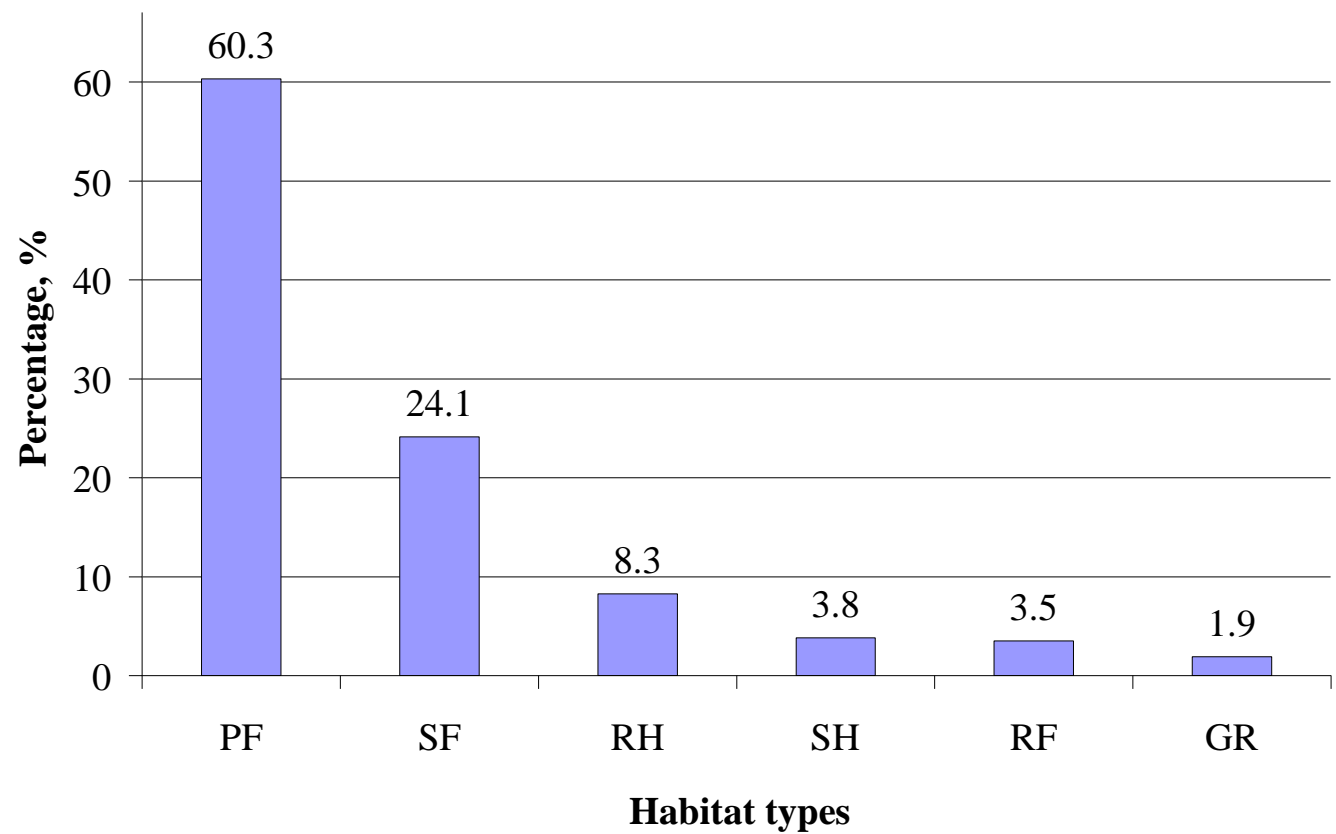

Fig. 4: The proportion of species number per habitat in the Behali Reserve Forest. Designations: PF - Pristine forests, SF - Secondary forests, RF - Rehabilitated forests, SH - Shrublands, RH - Riparian habitats, GR - Grasslands.

\section{Global IUCN Red List assessment of two endemic species}

Of 281 taxa in BRF, 58 plants species have global IUCN status [26]. Of these, 54 species are reported as Least Concern (Appendix), two species (Magnolia kingii (Dandy) Figlar and Magnolia griffithii Hook. f. \& Thomson) as Data Deficient, Elaeocarpus rugosus Roxb. ex G.Don as Vulnerable, and Aglaia edulis (Roxb.) Wall. as Near Threatened. In this paper, we carried out the global IUCN Red List of two more plant species on the basis of data obtained in North-east India (Fig. 5) during the present study.

\section{IUCN status of Chlorophytum assamicum}

Criterion B: There is a single location (Fig. 5C). AOO: $4 \mathrm{~km}^{2}$ calculated with a $2 \times 2$ $\mathrm{km}$-cell fixed grid by GeoCAT programme [1]. Decline in extent and quality of the habitat (iii) has been directly observed and expected to continue in the future.

Criterion D: Number of mature individuals is less than 50 .

Category: CR B1ab(iii)+2ab(iii); D.

IUCN status of Aristolochia assamica

Criterion B: AOO: $20 \mathrm{~km}^{2}$ calculated with a $2 \times 2 \mathrm{~km}$-cell fixed grid by GeoCAT [1]. EOO: $5769 \mathrm{~km}^{2}$ calculated with minimum convex hull by GeoCAT [1]. There are five location within the distribution range. Decline in extent and quality of the habitat (iii) has been directly observed and expected to continue in the future.

Category: EN B2ab(iii). 


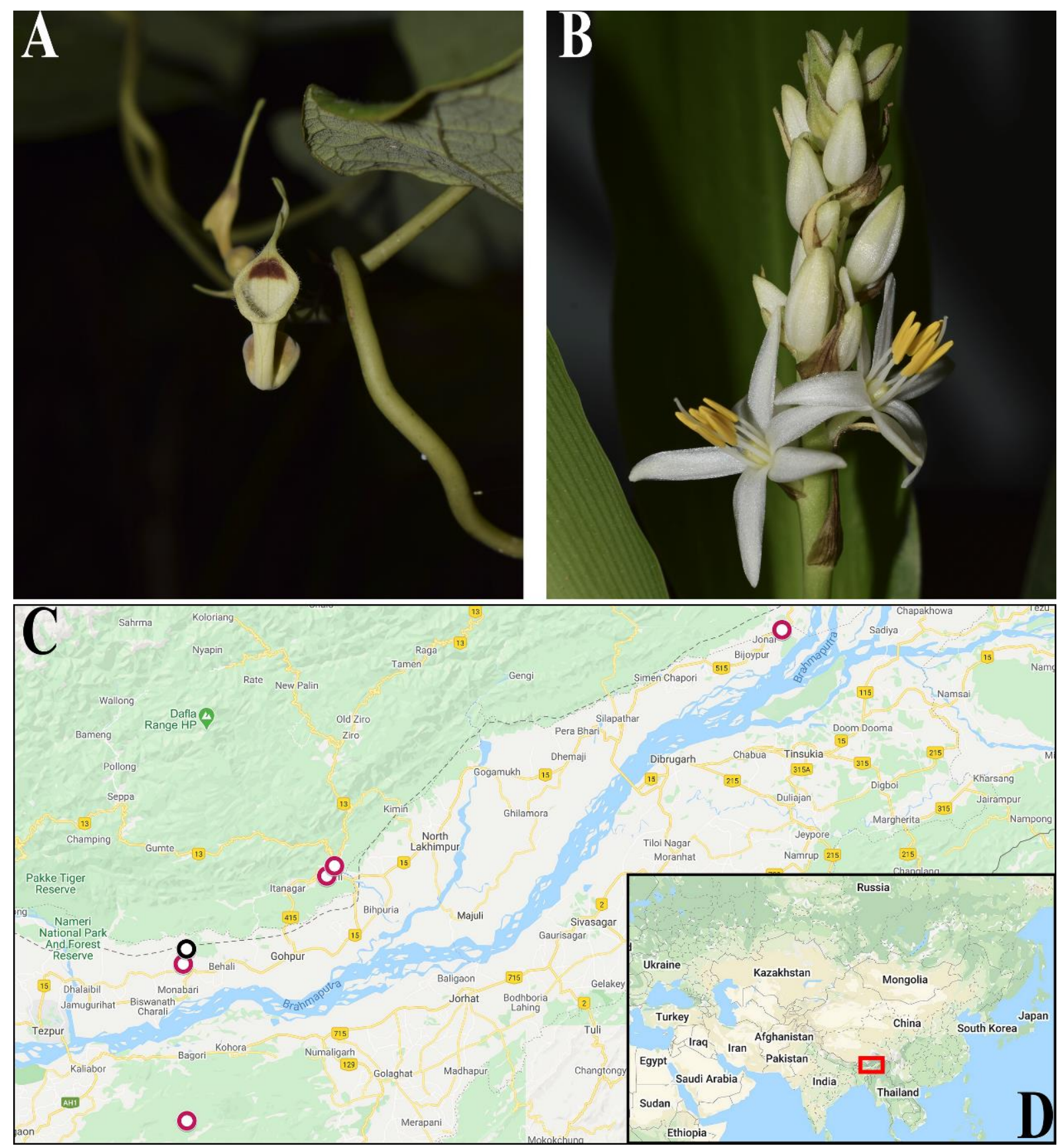

Fig. 5: A - Aristolochia assamica (red dots at map C); B - Chlorophytum assamicum (black dot at map C); their locations at map of Northeast India (C) and in South Asia (D).

\section{Discussion}

The data recorded indicates the presence of a rich floristic diversity in BRF as compared to other forests of Assam state. Though forest has been experiencing tremendous pressure for last few decades, through timber extraction, firewood collection, extension of human settlements, land encroachment and other such destructive activities, still some dense patches have been left in the core regions. The species list obtained is comparable to the studies of Majumdar \& Datta [34], Sarkar \& Devi [43], Borah et al. [6], Mipun et al. [36] in terms of number of species. At the same time, species richness in BRF is higher than Dutta \& Devi [18, 19], Bora \& Bhattacharya [5], Borogayary et al. [12], demonstrated in other forests of similar environment within the state of 
Assam. Concerning separate families, the predominant Orchidaceae makes the main contribution to epiphyte richness. Perhaps, the relatively lower proportion of orchids in taxonomic spectra is a result of underestimation of epiphytic floras.

In the studied flora, woody plants are predominated with the highest number of trees (104 taxa), vines (51 taxa) and shrubs (46 taxa). It is typical for tropical forests, where trees and shrubs make the most contribution to carbon stock [39]. This is in line with the highest species richness in pristine, secondary and rehabilitated forests. At the same time, we clearly found that the species richness decreased as both openness and disturbance level of a habitat increased. Our results are consistent with data that disturbances and plantation or rehabilitation of forests alter and decline the species composition in a plant community [33].

Results of the conducted IUCN Red List assessments of two endemic species, Aristolochia assamica and Chlorophytum assamicum, made it possible to continue the search and monitoring of their new locations and future re-assessment of IUCN status. Moreover, now, Critically Endangered $A$. assamica is the $14^{\text {th }}$ species in world and $1^{\text {st }}$ species in South and South-east Asia of the genus Aristolochia, which are estimated globally [26]. Finally, it is the $3^{\text {rd }}$ Critically Endangered Aristolochia species, while other ten species were previously assessed as Endangered (four taxa), Vulnerable (six taxa), Least Concern (one taxon). Chlorophytum assamicum is the $20^{\text {th }}$ species of the genus Chlorophytum estimated globally. It is the $3^{\text {rd }}$ globally assessed Chlorophytum species in both India and the whole of South and South-east Asia. Finally, C. assamicum is a $4^{\text {th }}$ Critically Endangered Chlorophytum species assessed at a global scale, while three more Chlorophytum taxa were globally estimated as Endangered, one species is Vulnerable, one species is Data Deficient, and, finally, 11 Chlorophytum have Least Concern status [26]. Taking into account the high level of species endemism in the tropics and, in particular, in India, we propose a need to estimate more number of plant species according to IUCN Red List categories and criteria in order to fill gaps in knowledge on threats to plant species in the world.

\section{Conclusions}

The BRF flora in the state of Assam demonstrates a high habitat diversity represented mainly by forests (pristine, secondary, rehabilitated), which reflects the predominance of woody plants in growth form spectrum. Although the present checklist is not an exhaustive inventory of the BRF (higher sampling effort is necessary to capture total local plant diversity), it serves as a starting point for future floristic and ecological studies in this and the nearest protected and managed areas in North-east Asia. Because during the present studies in BRF, three new species were described, one species was rediscovered after a century, as well as one new record obtained for the flora of Assam state [8,9]. And at the same time, several such new records of plant species for India and for science are highly possible in future. The global IUCN Red List assessment conducted of two endemic plant species highlights an opportunity to obtain further data on populations of other plant species and throws light on the need for conservation programmes for species recovery through populations built up in native habitats.

Acknowledgments: This research was supported by the Department of Botany, Rajiv Gandhi University, Arunachal Pradesh. The authors are thankful to Altafhusain Nadaf (University of Pune, India), Ankur Upadhaya (Rajiv Gandhi University, India), Biswajit Das (North Eastern Regional Institute of Science and Technology, India), David J. Middleton (Singapore Botanic Gardens, Singapore), Dilip Kumar Roy (Botanical Survey of India, India), Do Van Truong (Vietnam National Museum of Nature, Vietnam), Felipe Moreira (Rio de Janeiro, Brazil), Hussain Barbhuiya 
(Bhabha Atomic Research Centre, India), Hyeok Jae Choi (Changwon National University, Korea), J.R.I. Wood (University of Oxford, United Kingdom), Jana V Sudhakar (Dr. SRK Govt. Arts College, India), Jayanta Thakur (Gauhati University, India), Suhas Kadam Department of Environment \& Forests, India), Khyanjeet Gogoi (Regional Orchids Germplasm Conservation and Propagation Centre, India), Leonid Averyanov (Komarov Botanical Institute of RAS, Russia), Michele Rodda (Singapore Botanic Gardens, Singapore), Mark Hughes (Royal Botanic Garden Edinburgh, Scotland), Martin Calamander (Missouri Botanical Garden, Missouri),Mipun Puranjoy (BN College, India), Momang Taram (Rajiv Gandhi University, India), Munna Gorh (Biswanath College, India), N. Balachandran (French Institute of Pondicherry, India), Noriyuki Tanaka (Tokyo, Japan), Navendu Page (Indian Institute of Science, India), Nazir Ahmed Bhatt (North Eastern Hill University, India), Neelam Gap (North Eastern Regional Institute of Science and Technology, India), Nidhan Singh (IB College, India), Niku Das (Biswanath College, India), Padmaraj Gajurel (North Eastern Regional Institute of Science and Technology, India), Purna Tokbi (Biswanath, India), Rajeev Kumar Singh (Botanical Survey of India, India), Rajiv Barman (Biswanath College, India), Ritesh Kumar Choudhary (Agharkar Research Institute, India), Robert Dahanga (Tripura University, India), Rupam Bhujel (Gauhati University, India), Sabir Ahmed (Biswanath College, India), Sanjib Baruah (Bodoland University, India), Santanu Dey (Nagaland University, India), Santosh Nampy (Calicut University, India), Suman Kondha (Biswanath, India), Tapas Chakraborty (Kolkata, India), Todd J. Barkman (Western Michigan University, USA) for their cooperation in the field studies, identification, and drafting of this paper.

\section{REFERENCES}

1. Bachman, S., Moat, J., Hill, A.W., de la Torre, J., Scott, B., 2011, Supporting Red List threat assessments with GeoCAT: geospatial conservation assessment tool, ZooKeys, 150: 117-126. DOI: 10.3897/zookeys.150.2109

2. Baro, D., Bawri, A., Borthakur, S.K., 2019, Tolypanthus maclurei (Merrill) Danser (Loranthaceae): an addition to flora of India, from Manas National Park, Assam, National Academy Science Letters, 42 (5): 437-439. DOI: 10.1007/s40009-018-0776-1

3. Barooah, C., Ahmed, I., 2014, Plant diversity of Assam - a checklist of angiosperms and gymnosperms, Guwahati, Assam Science Technology and Environment Council.

4. Barooah, C., Mahanta, P.K., 2006, Aquatic angiosperms of Biswanath Chariali, Assam, Indian Journal of Forestry, 29 (3): 307-318.

5. Bora, A., Bhattacharyya, D., 2017, Phytodiversity of Barail Wildlife Sanctuary, Assam, India: field-based observations - I. Trees and lianas, Check List, 13 (6): 1037-1053. DOI: 10.15560/13.6.1037

6. Borah, N., Rabha, D., Athokpam, F.D., 2016, Tree species diversity in tropical forests of Barak valley in Assam, India, Tropical Plant Research, 3 (1): 1-9.

7. Borah, D., Kafley, P., Tangjang, S., Das, A.P., 2018, Population structure and conservation of endangered Citrus indica Yu.Tanaka (Rutaceae) in Behali Reserve Forest of Assam, India, Pleione, 12 (2): 181-186. DOI: 10.26679/Pleione.12.2.2018.181-186

8. Borah, D., Kafley, P., Das, A.P., Tangjang, S., Averynov, L., 2019a, Chlorophytum assamicum (Asparagaceae), a new species from Northeast India, Phytotaxa, 394 (1): 123-125.

DOI: 10.11646/phytotaxa.394.1.12

9. Borah, D., Taram, M., Das, A.P., Tangjang, S., Van Do, T., 2019b, Aristolochia assamica (Aristolochiaceae), a new species from the East Himalayas, Annales Botanici Fennici, 56 (4-6): 253-257.

DOI: $10.5735 / 085.056 .0410$

10. Borah, D., Taram, M., Tangjang, S., Upadhyaya, A., Tanaka, N., 2020a, Peliosanthes macrophylla var. assamensis (Asparagaceae), a new variety from Behali Reserve Forest in Assam, Northeast India, Blumea, 65 (2): 121-125. DOI: 10.3767/blumea.2020.65.02.05

11. Borah, D., Tanaka, N., Averyanov, L., Taram, M., Roy, D.K., 2020b, Rediscovery of Tupistra stoliczkana (Asparagaceae) in northeastern India and the identity of T. ashihoi, Phytotaxa, 443 (2): 207-210. DOI: 10.11646/phytotaxa.443.2.8

12. Borogayary, B., Das, A.K., Nath, A.J., 2017, Tree species composition and population structure of a secondary tropical evergreen forest in Cachar district, Assam, Journal of Environmental Biology, 39 (1): 67-71. DOI: 10.22438/jeb/39/1/MRN-487 
13. Brummitt, N.A., Bachman, S.P., Moat, J.F., 2008, Applications of the IUCN Red List: towards a global barometer for plant diversity, Endangered Species Research, 6: 127-135. DOI: 10.3354/esr00135

14. Chifundera, K.Z., 2019, Using diversity indices for identifying the priority sites for herpetofauna conservation in the Democratic Republic of the Congo, Nature Conservation Research, 4 (3): 13-33. DOI: 10.24189/ncr.2019.035

15. Devi, M., 1998, Herbaceous Angiosperms of Tezpur Sub-Division of Sonitpur District, Assam with reference to their Taxonomy and Scope utilization, PhD Thesis, Assam, Gauhati University.

16. Devi, M., Bhattacharyya, D., 2013, Panicum laxum (Poaceae): An addition to flora of India, Rheedea, 23 (2): 128-131.

17. Dirzo, R., Raven, P.H., 2003, Global state of biodiversity and loss, Annual Review of Environment and Resources, 28: 137-167. DOI: 10.1146/annurev.energy.28.050302.105532

18. Dutta, G., Devi, A., 2013a, Plant diversity and community structure in tropical moist deciduous sal (Shorea robusta Gaertn.) forest of Assam, Northeast India, Journal of Environmental and Applied Bioresearch, 1 (3): $1-4$.

19. Dutta, G., Devi, A., 2013b, Plant diversity, population structure and regeneration status in disturbed tropical forests in Assam, northeast India, Journal of Forestry Research, 24 (4): 715-720. DOI: 10.1007/s11676-0130409-y

20. Fenu, G., Bernardo, L., Calvo, R., Cortis, P., De Agostini, A., Gangale, C., Gargano, D., Gargano, M.L., Lussu, M., Medagli, P., Perrino, E.V., Sciandrello, S., Wagensommer, R.P., Orsenigo, S., 2019, Global and Regional IUCN Red List Assessments: 8, Italian Botanist, 8: 17-33. DOI: 10.3897/italianbotanist.8.47330

21. Gebremedihin, K.M., Birhane, E., Tadesse, T., Gbrewahid, H., 2018, Restoration of degraded drylands through exclosures enhancing woody species diversity and soil nutrients in the highlands of Tigray, Northern Ethiopia, Nature Conservation Research, 3 (1): 1-20. DOI: 10.24189/ncr.2018.001

22. Hooker, J.D., 1872-1897, The Flora of British India, vol. 1-7, London, Reeve and Company.

23. IUCN, 2012, IUCN Red List Categories and Criteria: Version 3.1, $2^{\text {nd }}$ edition, Gland and Cambridge, IUCN.

24. IUCN, 2013, Plant Growth Forms Classification Scheme. Version 1.0, available from https://nc.iucnredlist.org/redlist/content/attachment_files/nov_2013_plant_growth_forms_classification_sche me.pdf

25. IUCN, 2019, Guidelines for Using the IUCN Red List Categories and Criteria. Version 14, prepared by the Standards and Petitions Committee, available from http://www.iucnredlist.org/documents/RedListGuidelines.pdf

26. IUCN, 2020, The IUCN Red List of Threatened Species. Version 2020-1, available from https://www.iucnredlist.org

27. Jain, S.K., Rao, R.R., 1977, A handbook of field and herbarium methods, New Delhi, Today \& Tomorrow's Printers \& Publishers.

28. Jiang, Z., Zong, H., 2019, Reintroduction of the Przewalski's horse in China: status quo and outlook, Nature Conservation Research, 4 (Suppl.2): 15-22. DOI: 10.24189/ncr.2019.045

29. Kanjilal, U.N., Kanjilal, P.C., Das, A., Dey, R.N., Bor, N.L., 1934-1940, Flora of Assam, vol. 1-5, Shillong, Government of Assam.

30. Khapugin, A.A., 2020, A global systematic review on orchid data in Protected Areas, Nature Conservation Research, 5 (Suppl.1): 19-33. DOI: 10.24189/ncr.2020.019

31. Kushwaha, S.P.S., Hazarika, R., 2004, Assessment of habitat loss in Kameng and Sonitpur Elephant Reserves, Current Science, 87 (10): 1447-1453.

32. Linnik, E.V., Khapugin, A.A., 2020, Status of Tilia maximowicziana (Malvaceae s.1.) in Russia, Nature Conservation Research, 5 (2): 97-102. DOI: 10.24189/ncr.2020.007

33. Longworth, J.B., Williamson, G.B., 2018, Composition and diversity of woody plants in tree plantations versus secondary forests in Costa Rican lowlands, Tropical Conservation Science, 11: 1-13. DOI: $10.1177 / 1940082918773298$

34. Majumdar, K., Datta, B.K., 2014, A quantitative checklist of woody angiosperm diversity, population structure and habitat grouping in Trishna Wildlife Sanctuary of Tripura, northeast India, Check List, 10 (5): $976-996$. DOI: $10.15560 / 10.5 .976$

35. Ministry of Environment \& Forests, 2020, Protected Areas of India, available from http://www.wiienvis.nic.in/Database/Protect\%20ed_Area_854.aspx 
36. Mipun, P., Borah, D., Bhatt, N.A., Kumar, Y., 2019, Checklist for Phytodiversity of East Karbi Anglong Wildlife Sanctuary, Assam, Northeast India: Part I, Ambient Science, 6 (2). DOI: 10.21276/ambi.2019.06.2.aa02

37. Odyuo, N., Roy, D.K., Deori, C., Daimary, R., 2013, Ceratostylis radiata (Orchidaceae) - A new record to Indian flora, Rheedea, 23 (1): 43-45.

38. Orsenigo, S., Montagnani, C., Fenu, G., Gargano, D., Peruzzi, L., Abeli, T., Alessandrini, A., Bacchetta, G., Bartolucci, F., Bovio, M., Brullo, C., Brullo, S., Carta, A., Castello, M., Cogoni, D., Conti, F., Domina, G., Foggi, B., Gennai, M., Gigante, D., Iberite, M., Lasen, C., Magrini, S., Perrino, E.V., Prosser, F., Santangelo, A., Selvaggi, A., Stinca, A., Vagge, I., Villani, M., Wagensommer, R.P., Wilhalm, T., Tartaglini, N., Duprè, E., Blasi, C., Rossi, G., 2018, Red listing plants under full national responsibility: extinction risk and threats in the vascular flora endemic to Italy, Biological Conservation, 224: 213-222.

DOI: 10.1016/j.biocon.2018.05.030

39. Pennington, R.T., Lavin, M., Oliveira-Filho, A., 2009, Woody plant diversity, evolution, and ecology in the Tropics: perspectives from seasonally dry tropical forests, Annual Review of Ecology, Evolution, and Systematics, 40: 437-457. DOI: 10.1146/annurev.ecolsys.110308.120327

40. Poker, J., MacDicken, K., 2016, Tropical Forest Resources: Facts and Tables. In: Pancel, L., Köhl, M. (eds.) Tropical Forestry Handbook, Berlin, Heidelberg, Springer. DOI: 10.1007/978-3-642-54601-3_7

41. POWO, 2020, Plants of the World Online, facilitated by the Royal Botanic Gardens, Kew, available from http://www.plantsoftheworldonline.org/

42. Reddy, C.S., Jha, C.S., Diwakar, P.G., Dadhwal, V.K., 2015, Nationwide classification of forest types of India using remote sensing and GIS, Environmental Monitoring and Assessment, 187 (12): 777. DOI: 10.1007/s10661-015-4990-8

43. Sarkar, M., Devi, A., 2014, Assessment of diversity, population structure and regeneration status of tree species in Hollangapar Gibbon Wildlife Sanctuary, Assam, Northeast India, Tropical Plant Research, 1 (2): 26-36.

44. Sarma, P.K., Borah, R., Upadhaya, S., Dutta, S., Mahanta, G., 2009, A handbook of Behali Reserved Forest. Nature's Bonyopran, Assam, India.

45. Sarma, S.K., Borah, B., 2014, Phytosociological investigation of aquatic macrophytes of five wetlands of Sonitpur district of Assam, India, Annals of Biological Research, 5 (9): 38-45.

46. Singh, J.S., Chaturvedi, R.K., 2017, Diversity of ecosystem types in India: a Review, Proceedings of the Indian National Science Academy, 83 (2): 569-594. DOI: 10.16943/ptinsa/2017/49027

47. Silk, J.W.F., Arroyo-Rodríguez, V., Aiba, S.I., Alvarez-Loayza, P., Alves, L.F., Ashton, P., Balvanera, P., Bastian, M.L., Bellingham, P.J., van den Berg, E., Bernacci, L., da Conceição Bispo, P., Blanc, L., BöhningGaese, K., Boeckx, P., Bongers, F., Boyle, B., Bradford, M., Brearley, F.Q., Hockemba, M.B., Bunyavejchewin, S., Matos, D.C.L., Castillo-Santiago, M., Catharino, E.L.M., Chai, S.-L., Chen, Y., Colwell, R.K., Robin, C.L., Clark, C., Clark, D.B., Clark, D.A. et al. 2015. An estimate of the number of tropical tree species, Proceedings of the National Academy of Sciences, 112 (24): 7472-7477.

DOI: $10.1073 /$ pnas. 1423147112

48. Zanan, R.L., Nadaf, A.B., 2012, Pandanus martinianus (Pandanaceae), a new endemic species from northeastern India, Phytotaxa, 73: 1-7. DOI: 10.11646/phytotaxa.73.1.1

49. Zanan, R.L., Nadaf, A.B., 2013, Conservation Status of Indian Pandanaceae, American Journal of Plant Sciences, 4 (6A): 51-56. DOI: 10.4236/ajps.2013.46A008

\section{FLORA VASCULARĂ NATIVĂ DIN REZERVAȚIA PĂDUREA BEHALI (ASSAM, INDIA) CU EVALUAREA GLOBALĂ ÎN LISTELE ROȘII IUCN A DOUĂ SPECII ENDEMICE}

(Rezumat)

Investigarea florei şi faunei din ariile protejate aduce contribuții importante la cunoașterea statutului ecosistemelor naturale neadministrate. În perioada 2017-2019, am studiat flora vasculară din Rezervația Pădurea Behali (Assam, India). Rezervația are o suprafață de 140,16 km² și este o pădure semi-sempervirescentă. Am investigat compoziția taxonomică a florei şi diversitatea formelor de creștere a speciilor şi habitatelor. În plus, am evaluat două 
specii endemice (Aristolochia assamica și Chlorophytum assamicum) din punctul de vedere al criteriilor din Listelor Roșii IUCN. Rezultatele noastre au arătat că flora vasculară a Rezervației Pădurea Behali cuprinde 281 taxoni, cu 272 specii, o subspecie și opt varietăți. Acestea aparțin la 206 genuri și 79 familii. Printre acestea, Pandanus unguifer a fost menționat pentru prima dată în flora din Assam. În ceea ce privește spectrul formelor de creștere, au predominat arborii, lianele și tufărişurile, urmate de epifite, specii ierboase, geofite, anuale și parazite. Cel mai mare număr de specii de plante s-a înregistrat în zonele dense ale pădurii, în timp ce habitatele deschise s-au caracterizat prin cel mai mic număr de specii. Chlorophytum assamicum este evaluat ca CR B1ab(iii)+2ab(iii); D, iar Aristolochia assamica ca EN B2ab(iii). De asemenea, există o amenințare privind reducerea sau dispariția populațiilor acestor specii. Acest aspect este foarte important mai ales pentru Chlorophytum assamicum, reprezentat de o singură populație la nivel global. Cu siguranță studiile ulterioare ar putea pune în evidență un număr mai mare de specii floricole vasculare din Rezervația Pădurea Behali, precum și tendința de dezvoltare a populațiilor altor specii importante din punctul de vedere al categoriilor și criteriilor din Listele Roșii IUCN.

Received: 16.09.2020; Accepted: 19.11.2020. 